\title{
Optimized biometric system based iris-signature for human identification
}

\author{
Muthana Hachim Hamd \\ Computer Engineering Dept., Mustansiriyah University, Baghdad, Iraq \\ dr.muthana@uomustansiriyah.edu.iq
}

ARTICLE INFO

\section{Article history}

Received July 5, 2019

Revised August 9, 2019

Accepted October 29, 2019

Available online October 29, 2019

\section{Keywords}

Iris recognition

Fourier descriptors

Iris-Signature

Neural network

Optimization techniques

\begin{abstract}
This research aimed at comparing iris-signature techniques, namely the Sequential Technique (ST) and the Standard Deviation Technique (SDT). Both techniques were measured by Backpropagation (BP), Probabilistic, Radial basis function (RBF), and Euclidian distance (ED) classifiers. A biometric system-based iris is developed to identify 30 of CASIA-v1 and 10 subjects from the Real-iris datasets. Then, the proposed unimodal system uses Fourier descriptors to extract the iris features and represent them as an iris-signature graph. The 150 values of input machine vector were optimized to include only high-frequency coefficients of the irissignature, then the two optimization techniques are applied and compared. The first optimization (ST) selects sequentially new feature values with different lengths from the enrichment graph region that has rapid frequency changes. The second technique (SDT) chooses the high variance coefficients as a new feature of vectors based on the standard deviation formula. The results show that SDT achieved better recognition performance with the lowest vector-lengths, while Probabilistic and BP have the best accuracy.
\end{abstract}

This is an open access article under the CC-BY-SA license.

\section{Introduction}

In recent years, the biometrics recognition trait has become very important because of the growing number of frauds and the suffering of many traditional methods like password and personal identification number from some violations and rigging. To avoid breaches, biometric technologies are used to improve the security of human identification and verification. The iris is considered as one of the internal parts of the human body, and it is protected by cornea and eyelid. The reason for choosing iris because it cannot utilize after death because of the parts of the body would decay [1]. The iris contains many features such as contraction furrows, crypts, color, feature collagenous fibers, coronas, striations and serpentine vasculature [2] [3]. The iris recognition system works by capturing an iris image and a sample of this image, then transformed by using computational functions into a biometric machine vector or template. Then, the template is compared with other templates to find out the identity [4][5].

This paper presents iris verification using Fourier Descriptors (FDs) as a frequency domain feature extraction. The most important idea of the FDs is characterizing the contour for any object by frequency number contents for the whole shape. FDs acts to encode any two-dimensional object by converting its border into a complex number in the frequency domain. The iris feature extraction and template generation are depending on this transformation. Fourier coefficients are a small set of numbers that can be generated by relying on the frequency analysis. These coefficients describe the fine details of the shape, but it is not the noise that impacts on the boundary coordinate pixels. In the feature selection stage, the sufficient coefficients are chosen based on iris-signature and finding the standard deviation for selected 
vector sets. Three neural network classifiers and one Euclidean distance are applied to evaluate the classification results. The novelty of this work is the optimization methods that could maintain the biometric system accuracy rate and reducing the machine-vector size simultaneously [6] [7].

The ophthalmologist Frank Burch was the first person using an iris trait in human authentication in 1936. In 1987, Safir and Flom [1] were unsuccessful to implement Burch's idea to recognize persons depending on their iris. Daugman [8] [9] applied "Gabor filters" in phase-based iris recognition. The system showed excellent performance on various databases of a large number of images, where Hamming Distance (HD) was used in the matching phase between two codes. The system satisfied high accuracy and performance, which obtained $99.90 \%$ accuracy rate. In 2008, $\mathrm{Ng}$ et al. [10] suggested a segmentation method for iris recognition by using morphological operators and Circular Hough Transform (CHT). The proposed method rests on two search regions (right and left search regions) to detect the outer boundary of iris. The detection rate of the inner and outer of iris boundaries are $99.07 \%$ and $98.68 \%$, respectively.

In 2010, Koh et al. [11] suggested a method for iris segmentation based on an active contour model and CHT. The proposed method was tested using 100 iris images from CASIA v3.0 and achieved 99\% detection rate of the iris inner and outer boundary. In 2012, Das [12] proposed iris recognition approach using canny edge detection and $\mathrm{CHT}$ for localization of the iris and achieve $80 \%$ success rate using CASIA-v3.0-interval database. A modification was presented on Gabor wavelets for extracting a better discriminating feature vector. HD is utilized for matching; this approach attained a FRR of 5.26 and FAR of 4.72 .

In 2013, Kaur et al. [13] suggested two approaches for iris recognition, SVM and Phase-based procedure. SVM attained 19.8\% FRR and 0\% FAR while phase-based procedure achieved $0.01 \% \mathrm{FRR}$, 0.09\% FAR and 99.9\% overall recognition rate. In 2014, Jayalakshmi and Sundaresan [14] suggested the Fuzzy C-means algorithm and $\mathrm{K}$-means algorithm for iris segmentation. The two methods were implemented separately, and the accuracy rate was $98.20 \%$ with a low error rate. Kumar et al. [15] in 2016, proposed 2D discrete Cosine transform approach for iris feature extraction. Their proposal is applied to extract the most valuable iris features. Two publicly databases were considered for matching the iris patterns using HD method, IITD and CASIA v.4.0 databases. The suggested approach was attained $99.4 \%$ and $98.4 \%$ accuracy rate for CASIA-v4 and IITD databases, respectively.

In 2017, Hamd and Ahmed [16] applied Fourier descriptors and four classifiers for verification process; Radial Basis Function (RBF), Back-Propagation (BP), Euclidean Distance (ED), and Probabilistic. Gaikwad and Ali [17] used Wavelet decomposition and hamming distance for iris recognition. They find different accuracy rates with different dataset sizes for training and testing with the highest accuracy of them reach $89.03 \%$.

The main goal of this paper is to design a biometric system based on the iris-signature for human identification with maximum performance. The iris-signature graph could be obtained after applying the Fourier descriptors method for extracting the features in the frequency domain. The biometric system could be optimized if the machine vector is reduced to the lowest length with maintaining the accuracy at maximum value. So, two signature-based optimization techniques have been developed to select the maximum variant feature sets that could satisfy the maximum recognition rate for the system. Four classifiers: Backpropagation, Radial basis function, Probabilistic, and Euclidean distance are based on more competitive results. Also, 30 subjects from CASIA-V1 and 10 subjects from real-iris are considered as a system dataset.

The rest of the paper is organized as follows: Section 2 describes the proposed iris signature system which is containing iris acquisition, iris segmentation, localization, and recognition. The iris signature graph is represented after applying Fourier descriptors for extracting iris features; also, the two optimization techniques are explained and implemented for better performance. Section 3 tabulates the recognition results for the four classifiers and two datasets; it shows the accuracy rate for each method 
that is applied to 40 subjects. Eventually, section 4 discusses and concludes the highest accuracy result associated with its optimization method and classifier(s).

\section{Method}

In this work, the system is designed as a series of sequential processes starting from capturing an eye image until the identification process, as shown in Fig. 1. The biometric system components include image acquisition, iris and pupil segmentation, iris normalization, features extraction and selection, templet generating, and pattern matching. Back Propagation (BP ), Probabilistic, Radial Basis Function $(\mathrm{RBF})$, and Euclidian Distance (ED) are four classifiers that are applied for comparison and performance qualification. The first three classifiers are neural network-related classification.

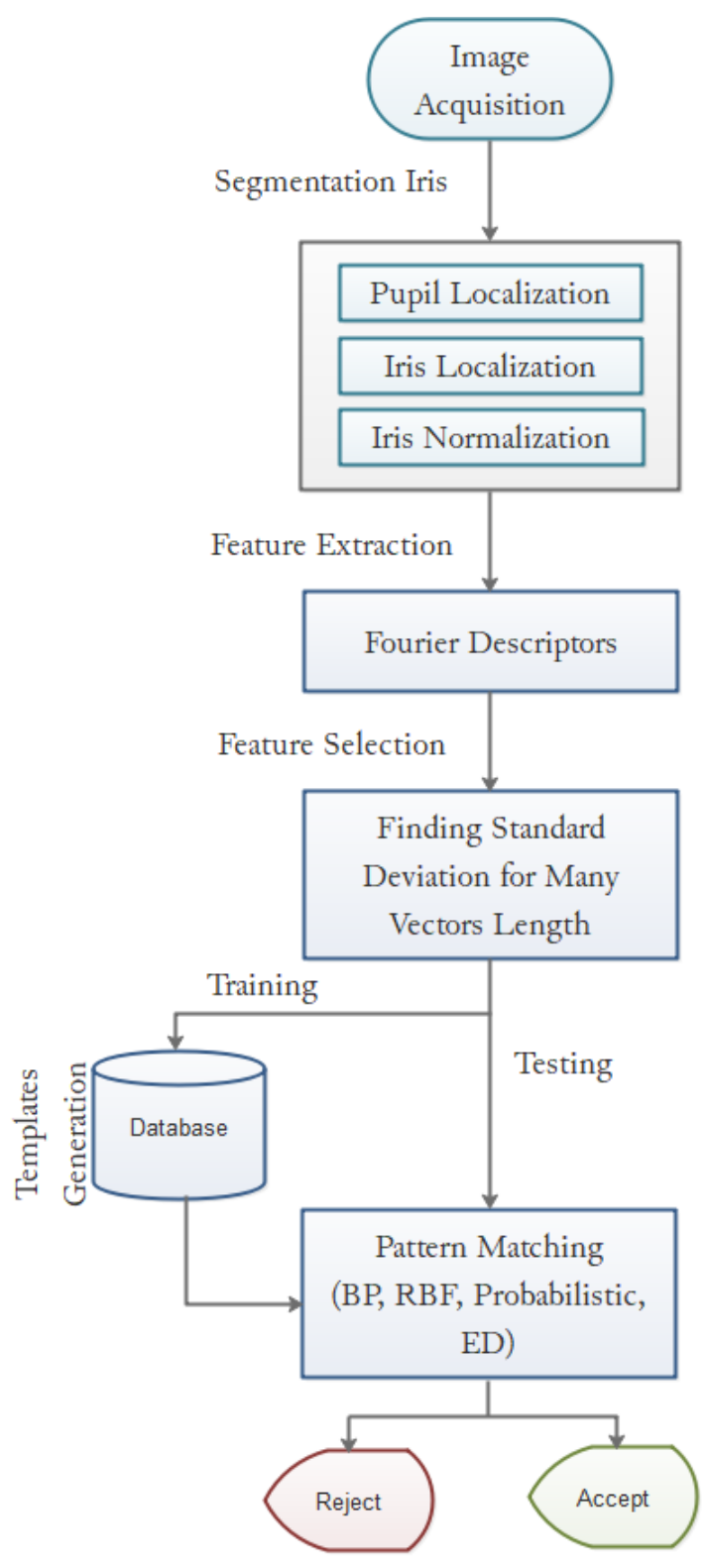

Fig. 1. The proposed iris recognition system [11]

\subsection{Image Acquisition}

The eye images are chosen from two databases; real-iris and the Chinese Academy of Sciences Institute of Automation (CASIA-v1) for the human identification system. The real-iris dataset of $400 \times$ 288 pixels is created from a distance of $7 \mathrm{~cm}$, while CASIA-v1 images are $320 \times 280$ pixels, as shown in Fig. 2. 


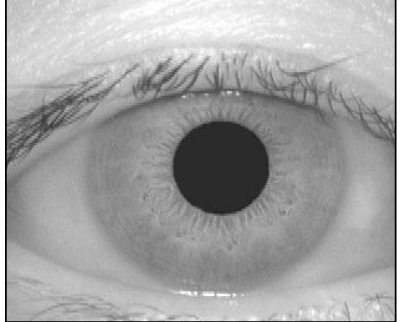

a) CASIA-v1

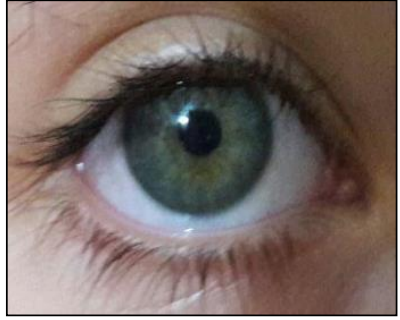

b) Real-iris

Fig. 2. Sample of irises [11]

\subsection{Iris Segmentation}

The segmentation stage comprises three necessary steps; pupil localization (which locates the center of the iris), localization and normalization step. These three steps have to be completed with high accuracy as they impact the verification operation [18]-[22].

1) Pupil localization. This stage is containing the following steps:

Step 1: converting the colored image to grayscale and applying a median filter on the resultant image.

Step 2: obtaining the binary image by converting the grayscale image using a suitable threshold as in (1).

$$
M(x, y)=\left\{\begin{array}{lr}
1 & f(x, y)=\tau \\
0 & f(x, y)<>\tau
\end{array}\right\}
$$

Step 3: remove the noise by applying morphological operations.

Step 4: applying the labeling algorithm for component connections

Step 5: Finding the pupil center $\left(P_{x}, P_{y}\right)$ by computing the summation of the horizontal and vertical vectors as in (2) and (3),

$$
\begin{aligned}
& \operatorname{hor}(x)=\sum_{1}^{x} \text { all rows } \\
& \operatorname{ver}(y)=\sum_{1}^{y} \text { all cols }
\end{aligned}
$$

Step 6: computing the maximum row and column vectors as in (4) and (5), then finding the radius of the pupil as in (6).

$$
\begin{aligned}
& P_{x}=\max (\text { hor }) \\
& P_{y}=\max (\text { ver }) \\
& r_{p}=(\max (\text { hor })-\min (\text { hor })) / 2
\end{aligned}
$$

where $x$ is is a number of rows, and $y$ is a number of columns.

2) Iris localization. This stage applies Canny edge detectors and Circular Hough Transform (CHT) for iris localization. So, the boundaries of iris contour are detected, and the complete circle shape is founded by the CHT method. Determining the center and radius of the iris is explained in $(7,8,9$, 10,11 and 12) [23]-[25].

3) Iris normalization. The normalization step is applying when there is a demand for image invariant against skewing or extension. Moreover, it minimizes the distortion resultant from the pupil movement effect. The normalization process starts by applying Daugman's rubber sheet method and transforming the circle iris region to a rectangular region. This method computes the polar form ( $\mathrm{r}$, $\theta$ ) from cartesian coordinates. The notion of polar form is to assign the radius ( $r$ ) to 20 pixels, and 
240 pixels along the angle $(\theta)$ to generate an unwrapped strip size of 4800 pixels $(20 \times 240)$. The transforming equations from Cartesian form to another polar form are explained as follows [3]:

$$
\begin{gathered}
X(r, \theta)=(1-r) * X_{P}(\theta)+r * X_{i}(\theta) \\
Y(r, \theta)=(1-r) * Y_{P}(\theta)+r * Y_{i}(\theta) \\
X_{p}(\theta)=X_{p 0}(\theta)+r_{p} * \cos (\theta) \\
Y_{p}(\theta)=Y_{p 0}(\theta)+r_{p} * \sin (\theta) \\
X_{i}(\theta)=X_{i 0}(\theta)+r_{i} * \cos (\theta) \\
Y_{i}(\theta)=Y_{i 0}(\theta)+r_{i} * \sin (\theta)
\end{gathered}
$$

where $\left(X_{p}, Y_{p}\right)$ indicates the pupil center, $\left(X_{i}, Y_{i}\right)$ indicates the iris center, $r_{p}$ and $r_{i}$ represent the radius of the pupil and iris. After the iris normalization step, the median filter is applied on iris rectangular to remove the effect of eyelid and eyelashes. Fig. 3 shows the results of the iris segmentation stage.

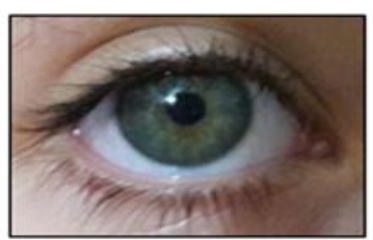

(a) Original Image

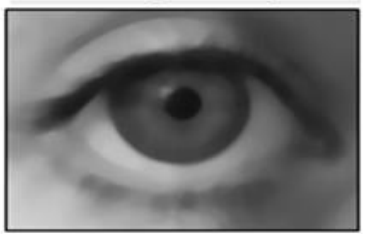

(c) Apply median filter

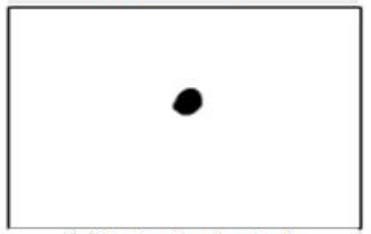

(e) Morphological Operation

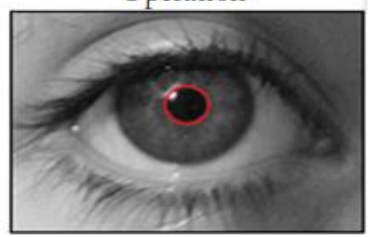

(g) Pupil localization

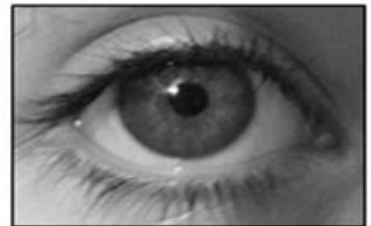

(b) Gray Image

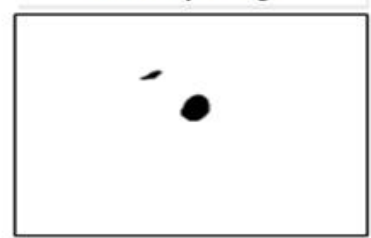

(d) Binary Image

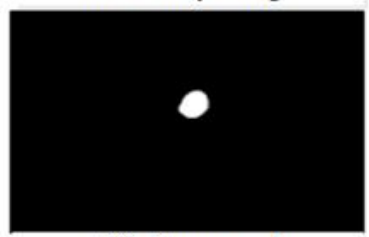

(f) Connected component

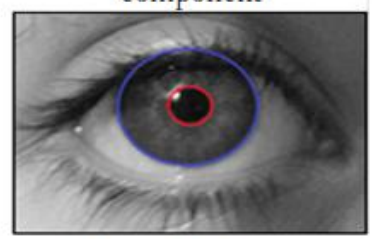

(h) Iris localization

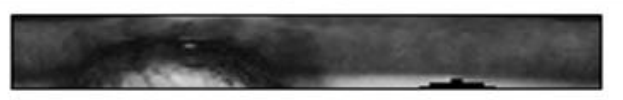

(i) Iris normalization

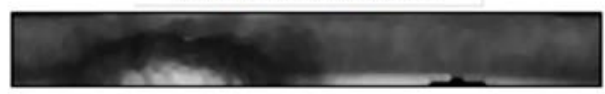

(j) De-noise Iris image

Fig. 3. Iris/pupil segmentation process and normalization 


\subsection{Feature Extraction Using Fourier Descriptors}

Many approaches are applied for feature extraction and template generation, for example, Gabor wavelet; spatial filters; local variance; zero-crossing wavelet; and Local Texture Pattern (LTP). Fourier Descriptor is used to create the feature vectors by transforming the iris description vector into the frequency domain. The fine details of iris are existing in the high frequencies coefficients while the lowfrequency coefficients belong to the general object information [26]. A large number of generated coefficients can be represented in the iris-signature graph as shown in Fig. 4. According to the classifier basic-operation, the sufficient-coefficient features are enough to describe the object shape based on a statistical method [27]. The FDs procedure is started by converting a rectangular image to binary, counting boundary points, and choosing a coefficient number $(\mathrm{N})$. Then, calculate centroid distance as in (13), compute Fourier descriptors values as in (13) and (14), calculate the FDn as in (15), and normalize it by dividing on the maximum (DC) value as in (16).

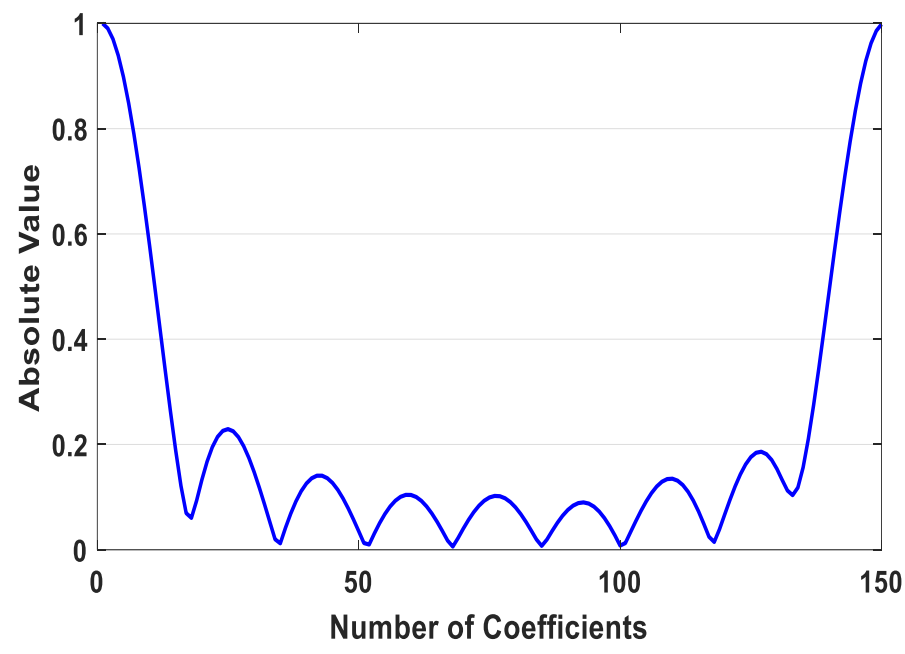

Fig. 4. Iris-Signature [11]

The FDs of pre-processed iris image $(4 \times 128)$ creates a relatively long vector with 150 coefficients, so a set of small vectors are required to include the significant features only. Those new vector-sets will be computed or chosen as explained in the next section. The implementation affected to the biometric system optimization. In other words, the simple optimization process is satisfying when it minimizes the machine vector length to accelerate the training and matching time at the highest accuracy rate [28] [29].

$$
\begin{aligned}
& \mathrm{r}(\mathrm{t})=\sqrt{\left[x(t)-x_{C}\right]^{2}+\left[y(t)-y_{c}\right]^{2}} \\
& x_{c}=\frac{1}{N} \sum_{t=0}^{N-1} x(t) \quad, \quad y_{C}=\frac{1}{N} \sum_{t=0}^{N-1} y(t) \\
& F D_{n}=\frac{1}{N} \sum_{t=0}^{N-1} r(t) * \exp \left(\frac{-2 j \pi n t}{N}\right) \\
& f=\frac{\left|F D_{1}\right|}{\left|F D_{0}\right|}, \frac{\left|F D_{2}\right|}{\left|F D_{0}\right|}, \ldots \cdots \frac{\left|F D_{N / 2}\right|}{\left|F D_{0}\right|}
\end{aligned}
$$

\subsection{Optimization Methods}

Two techniques which considered for selecting significant features to represent the input machine vector are 1) Sequential Technique (ST), and 2) Standard Deviation Technique (SDT). 
The ST chooses a new representative feature from the enriched iris-signature graph (the most-right or most-left area) and excludes the rest coefficients (Fig. 4). These enriched regions have a positive or negative gradient, and the feature values of these regions are very suitable for linear classifiers-based discrimination function. The SDT measures how the values are spreading which reflect the relation among coefficients. Then, the high-frequency coefficients are chosen depending on their highest standard deviation value, while the low standard deviation values are concentrated in the middle of the iris graph. The standard deviation formula is calculated as in (17) and the new vector set is listed in Table 1. Fig. 5 explains how these new vector lengths $(10,20,30,50,100)$ are reconstructed from the iris graph using the SDT technique. The SDT has improved the system performance by reducing the runtime and connection complexity. For example, when the input machine vector length is reduced from 150 to only 20 values, the number of neurons in the input layer will be reduced by the ratio of $86.67 \%$.

$$
S D=\sqrt{\frac{1}{m} \sum_{i=1}^{m}\left(x_{i}-\mu\right)^{2}}
$$

where $\left(x_{i}, \mu\right)$ represents the input vector and its associated mean value, respectively, $m$, is the vector length value.

From Table 1, the SDT for the excluded coefficients which are usually concentrated in the middle of an iris-signature graph is very low $(0.0029,0.0039, \ldots ., 0.0235)$. It respects the optimized values that have been taken from the enrichment graph area (mostly right and mostly left).

Table 1. The Optimal Five Vectors Using SDT Technique

\begin{tabular}{c|ccc|}
\hline \multicolumn{2}{c}{ Selected Coefficients } & \multicolumn{2}{c}{ Excluded Area } \\
\hline Optimized vector length & SDT & Excluded coefficients range & SDT \\
\hline 100 & 0.1747 & $50 \rightarrow 100$ & 0.0029 \\
\hline 50 & 0.2349 & $25 \rightarrow 125$ & 0.0039 \\
30 & 0.2838 & $15 \rightarrow 135$ & 0.0051 \\
\hline 20 & 0.3212 & $10 \rightarrow 140$ & 0.0086 \\
\hline 10 & 0.3689 & $5 \rightarrow 145$ & 0.0235 \\
\hline
\end{tabular}

\subsection{Training and Matching}

In this stage, 40 subjects from two databases are used for the verification process: 30 persons from the CASIA-v1 database and 10 persons from the real-iris dataset. Each person has 4 images ( 3 training images and one for testing). The four classifiers are applied for comparing the classification results where three of them are neural networks and one is distance measurement. The BP is run with 4 layers: 1 input layer, 2 hidden layers and an output layer. The two hidden layers containing 60 neurons i.e. $60 \times 2=120$ neurons. The RBF and Probabilistic neural network containing 3 layers: one input layer, one hidden layer, and an output layer, the hidden layer containing 90 neurons [30]. The ED classifier calculates the distance between the testing machine vector and the stored training vectors, as in (18). Then, the distance is checked; if it is less than a threshold value, it means belongs to the same class; otherwise, it belongs to a different one.

$$
d(X, Y)=\sqrt{\sum_{i=1}^{n}\left(X_{i}-Y_{i}\right)^{2}}
$$




\section{Results and Discussion}

The proposed system was implemented on two databases using two groups of input machine-vectors. The first group utilized the whole 150 iris coefficients (with no optimization), while the vector lengths of the second group are submitted to the optimization rules. The rules generated a set of different vector lengths: 10, 20, 30, 50 and 100 values, which belong to the ST and SDT techniques equally.

The ST optimization, as its name refers, selects the new vector values from the enrichment region of the iris-signature graph in Fig. 4 sequentially. Those five different lengths of optimized vectors are submitted to four different classification methods as explained in Table 2. The recognition rate of the real-iris is very interested and it could satisfy advanced accuracy rates for all classifiers, but the only $B P$ classifier could maintain the maximum accuracy rate $100 \%$ using only 100 vector values (optimized). Table 3 tabulates the ST recognition rates with the CASIA-V1 dataset. It is noticing that the ST optimization has kept a kind of performance stability for the biometric system for all classifiers for the vector length reduction. However, the Probabilistic neural network achieved a valuable recognition rate of $86.67 \%$, with only 20 optimized vector values.

Table 2. ST and SDT Performance using 10 subjects from Real-iris Dataset

\begin{tabular}{|c|c|c|c|c|c|c|c|}
\hline \multirow{3}{*}{$\begin{array}{l}\text { Optimization } \\
\text { Methods }\end{array}$} & \multirow{3}{*}{ Classifiers } & \multicolumn{6}{|c|}{ Accuracy rate (\%) } \\
\hline & & \multicolumn{5}{|c|}{ Optimized Vector Sets } & \multirow{2}{*}{$\begin{array}{c}\text { Original Vector Length } \\
150\end{array}$} \\
\hline & & 10 & 20 & 30 & 50 & 100 & \\
\hline \multirow{4}{*}{$\mathrm{ST}$} & $B P$ & 70 & 80 & 90 & 90 & 100 & 100 \\
\hline & $R B F$ & 80 & 80 & 80 & 80 & 90 & 100 \\
\hline & Probabilistic & 90 & 90 & 90 & 90 & 90 & 100 \\
\hline & $E D$ & 90 & 90 & 80 & 80 & 80 & 100 \\
\hline \multirow{4}{*}{ SDT } & $B P$ & 80 & 90 & 90 & 100 & 100 & 100 \\
\hline & $R B F$ & 90 & 90 & 90 & 100 & 90 & 100 \\
\hline & Probabilistic & 90 & 100 & 100 & 100 & 100 & 100 \\
\hline & $E D$ & 90 & 100 & 100 & 100 & 100 & 100 \\
\hline
\end{tabular}

Table 3. ST and SDT Performance using 30 subjects from CASIA-V1 Dataset

\begin{tabular}{|c|c|c|c|c|c|c|c|}
\hline \multirow{3}{*}{$\begin{array}{l}\text { Optimization } \\
\text { Methods }\end{array}$} & \multirow{3}{*}{ Classifiers } & \multicolumn{6}{|c|}{ Accuracy rate (\%) } \\
\hline & & \multicolumn{5}{|c|}{ Optimized Vector Sets } & \multirow{2}{*}{$\begin{array}{c}\text { Original Vector Length } \\
150\end{array}$} \\
\hline & & 10 & 20 & 30 & 50 & 100 & \\
\hline \multirow{4}{*}{ ST } & $B P$ & 80 & 73.33 & 86.67 & 83.33 & 80 & 96.67 \\
\hline & $R B F$ & 66.67 & 76.67 & 76.67 & 76.67 & 73.33 & 83.33 \\
\hline & Probabilistic & 83.33 & 86.67 & 83.33 & 83.67 & 83.33 & 86.67 \\
\hline & $E D$ & 66.67 & 70 & 70 & 70 & 70 & 96.67 \\
\hline \multirow{4}{*}{ SDT } & $B P$ & 86.67 & 93.33 & 90 & 96.67 & 100 & 96.67 \\
\hline & $R B F$ & 90 & 90 & 83.33 & 83.33 & 83.33 & 83.33 \\
\hline & Probabilistic & 80 & 86.67 & 90 & 90 & 86.67 & 86.67 \\
\hline & $E D$ & 90 & 93.33 & 93.33 & 93.33 & 96.67 & 96.67 \\
\hline
\end{tabular}


The five different lengths of machine vector values for the SDT optimization process are illustrated in Fig. 5(a-f). The optimization technique selects the new vector values from the most right and most left of the iris-signature graph. It also adopted the standard deviation formula as evident for increasing the discrimination between vector values. Table 2 shows, in detail, the real-iris recognition rates using four classifiers and six different vector sizes.

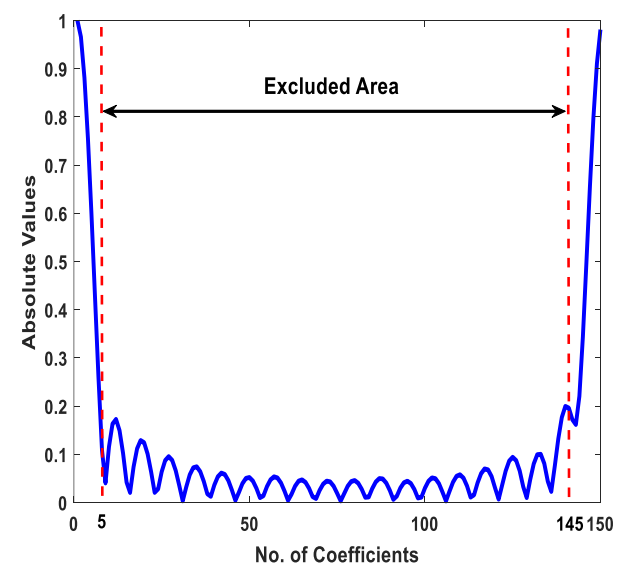

(a) Optimum 10 values

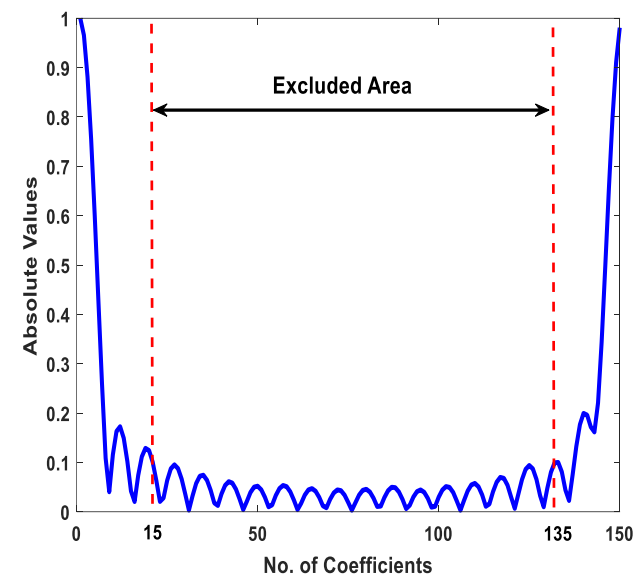

(c) Optimum 30 values

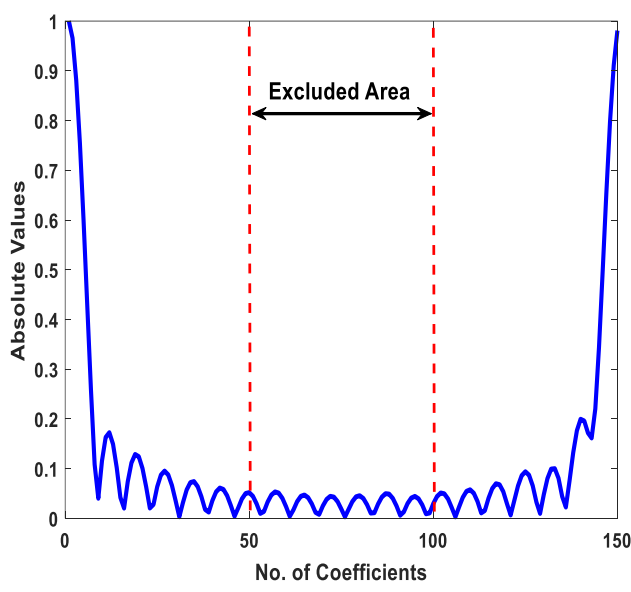

(e) Optimum 100 values

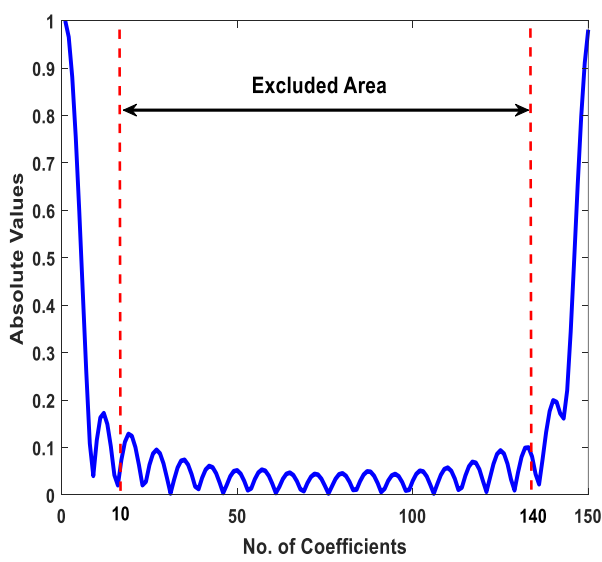

(b) Optimum 20 values

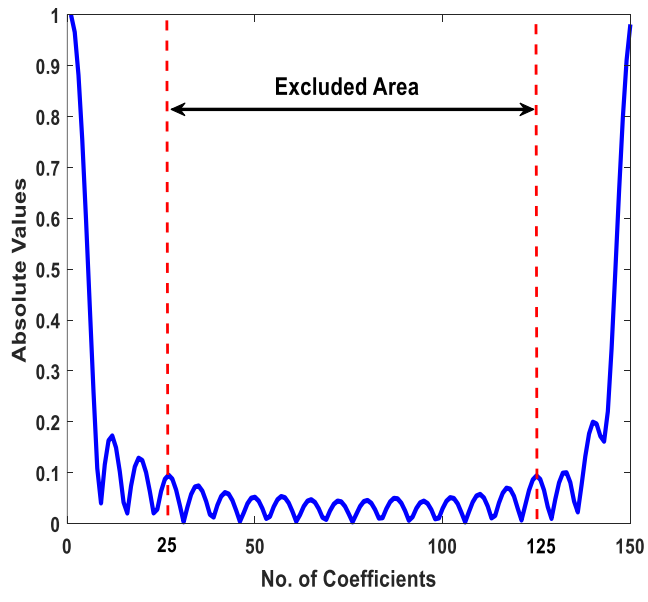

(d) Optimum 50 values

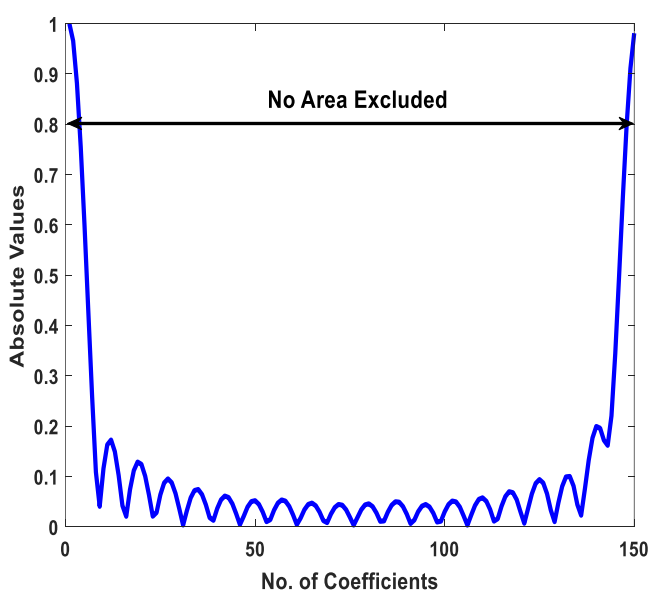

(f) Original 150 values

Fig. 5. Real-iris optimizing operations using standard deviation technique 
The $B P$, Probabilistic, and $E D$ have been recorded as the best classifiers that maintain the original accuracy rate in contrast to the lowest vector length. So, for the real-iris dataset, the SDT optimization was very effective in improving the accuracy rates with the lowest vector size. Hereby, the BP and RBF achieved a maximum recognition rate of $100 \%$ with an optimized vector length of 50 values only. The optimum classification results of the SDT technique are satisfied with the Probabilistic and ED classifier, where they obtained a 100\% accuracy rate with a vector length of 20 values only.

The story with the CASIA-V1 dataset is different, were some classifiers, such as the BP, Probabilistic and $\mathrm{RBF}$ recorded new significant recognition rates as shown in Table 3. The $\boldsymbol{B P}$ satisfied $100 \%$ recognition rate with 50 values vector length, while the $\mathrm{RBF}$ achieved a $90 \%$ recognition rate with a minimum vector length of 10 values only. Finally, $\boldsymbol{B P}$ and Probabilistic are the two most scored classifiers that operate with ST and SDT techniques efficiently. The Probabilistic satisfied $90 \%$ and $86.67 \%$ accuracy rate with vector lengths of 30 and 20 values, respectively.

For more evaluation of the biometric system, Table 4 tabulated the run-time comparison of the two databases and four classifiers. The best run-time results are satisfied with the Probabilistic classifier. It ran the CASIA-V1 dataset in $(3.76,2.26)$ second with a vector length of 150 and 20 values (SDT), respectively, while Probabilistic takes a shorter time $(2.568,0.488)$ second to run the real-iris dataset with same vector length, respectively. The relatively shortest run-time of the real-iris dataset belongs to the number of subjects (10) comparison to the CASIA-V1 (30) subject.

Table 4. Recognition Time Comparision

\begin{tabular}{cccc}
\hline Databases & Classifiers & $\begin{array}{c}\text { SDT run-time (sec) for } \\
\text { vector length =150 }\end{array}$ & $\begin{array}{c}\text { SDT run-time (sec) for } \\
\text { vector length }=\mathbf{2 0}\end{array}$ \\
\hline & BP & 120.24 & 65.351 \\
CASIA-v1 & RBF & 11.556 & 6.361 \\
& Probabilistic & 3.762 & 2.265 \\
& ED & 22.286 & 3.972 \\
Real-iris & BP & 40.573 & 36.598 \\
& RBF & 6.138 & 0.531 \\
& Probabilistic & 2.568 & 0.488 \\
& ED & 3.804 & 1.376 \\
\hline
\end{tabular}

\section{Conclusion}

A unimodal iris recognition system was developed to recognize forty persons taken from two databases. Fourier descriptor was based as a frequency-domain feature extractor to generate and represent 150 coefficients in the iris-signature graph. The performance of those 150 coefficients achieved $100 \%$ and $96.67 \%$ maximum recognition rate for real-iris and CASIA-V1 datasets, respectively. Two optimization techniques: ST and SDT were also applied to save time and reduce the computations and connection cost in the classifiers. The RBF attained a $90 \%$ accuracy rate with only 10 vector-length values, while the $\mathrm{BP}$ satisfied a maximum accuracy of $100 \%$ with 50 values. The most important was the Probabilistic that has minimum execution times among all other classifiers. It spent only 3.762 seconds for CASIA-V1 and 2.568 seconds for real-iris databases, while the BP spent 120.24 seconds and 40.573 seconds for the same databases and vector-length value (150). According to these experimental results, the SDT had better performance than ST, where the Probabilistic and BP methods are nominated to be the best classifiers that maintained or enhanced the original accuracy rate at lower vector lengths. Probabilistic, however, advanced BP method as it required less execution time, for example, for 150 coefficients; Probabilistic was 32 times faster than BP in recognizing 30 subjects from CASIA-V1 database.

\section{Acknowledgment}

My sincere thanks and gratitude to the University of Al Mustansiriyah, Baghdad, Iraq. 


\section{References}

[1] A. K. Jain, A. A. Ross, and K. Nandakumar, Introduction to Biometrics, 2011, doi: 10.1007/978-0-38777326-1.

[2] R. M. Bodade and S. N. Talbar, Iris Analysis for Biometric Recognition Systems, 2014, doi: 10.1007/978-81322-1853-1.

[3] M. H. Hamd and S. K. Ahmed, "Biometric System Design for Iris Recognition Using Intelligent Algorithms," Int. J. Mod. Educ. Comput. Sci., vol. 10, no. 3, pp. 9-16, Mar. 2018, doi: 10.5815/ijmecs.2018.03.02.

[4] P. R. Nalla and A. Kumar, "Toward More Accurate Iris Recognition Using Cross-Spectral Matching," IEEE Trans. Image Process., vol. 26, no. 1, pp. 208-221, Jan. 2017, doi: 10.1109/TIP.2016.2616281.

[5] M. H. Hamd and M. Y. Mohammed, "Multimodal Biometric System based Face-Iris Feature Level Fusion," Int. J. Mod. Educ. Comput. Sci., vol. 11, no. 5, pp. 1-9, May 2019, doi: 10.5815/ijmecs.2019.05.01.

[6] R. W. Ives, "Use of one-dimensional iris signatures to rank iris pattern similarities," Opt. Eng., vol. 45, no. 3, p. 037201, Mar. 2006, doi: 10.1117/1.2181140.

[7] R. M. da Costa and A. Gonzaga, "Dynamic Features for Iris Recognition," IEEE Trans. Syst. Man, Cybern. Part B, vol. 42, no. 4, pp. 1072-1082, Aug. 2012, doi: 10.1109/TSMCB.2012.2186125.

[8] J. G. Daugman, "High confidence visual recognition of persons by a test of statistical independence," IEEE Trans. Pattern Anal. Mach. Intell., vol. 15, no. 11, pp. 1148-1161, 1993, doi: 10.1109/34.244676.

[9] J. Daugman, "How Iris Recognition Works," IEEE Trans. Circuits Syst. Video Technol., vol. 14, no. 1, pp. 21-30, Jan. 2004, doi: 10.1109/TCSVT.2003.818350.

[10] R. Y. F. Ng, Yong Haur Tay, and Kai Ming Mok, "An effective segmentation method for iris recognition system," in 5th International Conference on Visual Information Engineering (VIE 2008), 2008, pp. 548-553, doi: 10.1049/сp:20080375.

[11] J. Koh, V. Govindaraju, and V. Chaudhary, "A Robust Iris Localization Method Using an Active Contour Model and Hough Transform," in 2010 20th International Conference on Pattern Recognition, 2010, pp. 28522856, doi: 10.1109/ICPR.2010.699.

[12] A. Das, "Recognition of Human Iris Patterns," National Institute of Technology, Rourkela, 2012, available at: http://ethesis.nitrkl.ac.in/3635/.

[13] G. Kaur, D. Kaur, and D. Singh, "Study of Two Different Methods for Iris Recognition Support Vector Machine and Phase Based Method," Int. J. Comput. Eng. Res., vol. 3, no. 4, pp. 88-94, 2013, available at : Google Scholar.

[14] S. Jayalakshmi and M. Sundaresan, "A Study of Iris Segmentation Methods using Fuzzy C-Means and KMeans Clustering Algorithm," Int. J. Comput. Appl., vol. 85, no. 11, pp. 1-5, Jan. 2014, doi: 10.5120/148823316.

[15] A. Kumar, A. Potnis, and A. Singh, "Iris recognition and feature extraction in iris recognition system by employing 2D DCT," IRJET Int. Res. Comput. Sci. Softw. Eng. Tecbnol., vol. 3, no. 12, pp. 503-510, 2016, available at : Google Scholar.

[16] M. Hamd, and Samah Ahmed, "Fourier Descriptors for Iris Recognition," Int. J. Comput. Digit. Syst., vol. 6, no. 5, pp. 285-291, Sep. 2017, doi: 10.12785/IJCDS/060507.

[17] A. T. and M. M., "Iris Feature Extraction and Matching by using Wavelet Decomposition and Hamming Distance," Int. J. Comput. Appl., vol. 158, no. 4, pp. 43-47, Jan. 2017, doi: 10.5120/ijca2017912793.

[18] D. Zhang, G. Lu, and others, "A comparative study on shape retrieval using Fourier descriptors with different shape signatures," in Proc. of international conference on intelligent multimedia and distance education (ICIMADE01), 2001, pp. 1-9, available at : Google Scholar.

[19] S. Viriri and J. R. Tapamo, "Integrating Iris and Signature Traits for Personal Authentication Using UserSpecificWeighting," Sensors, vol. 12, no. 4, pp. 4324-4338, Mar. 2012, doi: 10.3390/s120404324.

[20] P. De and D. Ghoshal, "Recognition of Non Circular Iris Pattern of the Goat by Structural, Statistical and Fourier Descriptors,” Procedia Comput. Sci., vol. 89, pp. 845-849, 2016, doi: 10.1016/j.procs.2016.06.070. 
[21] A. S. Falohun, E. O. Omidiora, A. O. Akanni, and A. S. Awofadeju, "Remote Access Control System using Iris Signature," in Proceedings of the World Congress on Engineering and Computer Science, 2013, vol. 1, available at : Google Scholar.

[22] G. Zhang, Z. Ma, L. Niu, and C. Zhang, "Modified Fourier descriptor for shape feature extraction," J. Cent. South Univ., vol. 19, no. 2, pp. 488-495, Feb. 2012, doi: 10.1007/s11771-012-1030-5.

[23] K. Misztal, E. Saeed, J. Tabor, and K. Saeed, "Iris Pattern Recognition with a New Mathematical Model to Its Rotation Detection,” 2012, pp. 43-65, doi: 10.1007/978-1-4614-5608-7_3.

[24] H. Mehrotra, P. K. Sa, and B. Majhi, "Fast segmentation and adaptive SURF descriptor for iris recognition," Math. Comput. Model., vol. 58, no. 1-2, pp. 132-146, Jul. 2013, doi: 10.1016/j.mcm.2012.06.034.

[25] F. N. Sibai, H. I. Hosani, R. M. Naqbi, S. Dhanhani, and S. Shehhi, "Iris recognition using artificial neural networks," Expert Syst. Appl., 2011, doi: 10.1016/j.eswa.2010.11.029.

[26] A. Amanatiadis, V. G. Kaburlasos, A. Gasteratos, and S. E. Papadakis, "Evaluation of shape descriptors for shape-based image retrieval," IET Image Process., vol. 5, no. 5, p. 493, 2011, doi: 10.1049/iet-ipr.2009.0246.

[27] B. Esmael, A. Arnaout, R. K. Fruhwirth, and G. Thonhauser, "A statistical feature-based approach for operations recognition in drilling time series," Int. J. Comput. Inf. Syst. Ind. Manag. Appl., vol. 5, pp. 454461, 2015, available at : Google Scholar.

[28] M. LEI, "Medical Image Enhancement Using Fourier Descriptors and Hybrid Filters," 2009, pp. 59-69, doi: 10.1016/B978-012373904-9.50011-8.

[29] Z. He, T. Tan, and Z. Sun, "Iris localization via pulling and pushing," in Proceedings - International Conference on Pattern Recognition, 2006, doi: 10.1109/ICPR.2006.724.

[30] I. G. P. S. Wijaya, I. B. K. Widiartha, K. Uchimura, M. S. Iqbal, and A. Y. Husodo, "Fast pornographic image recognition using compact holistic features and multi-layer neural network," Int. J. Adv. Intell. Informatics, vol. 5, no. 2, p. 89, May 2019, doi: 10.26555/ijain.v5i2.268. 